\title{
Initial Experience of Using Polymyxin B Hemoperfusion in Abdominal Septic Shock: Things to Consider for Better Outcome
}

\author{
Young Jun Park a, Eun Young Kim b,* \\ a Department of Surgery, Seoul St. Mary's Hospital, College of Medicine, The Catholic University of Korea, Seoul, Korea \\ ${ }^{b}$ Division of Trauma and Surgical Critical Care, Department of Surgery, Seoul St. Mary's Hospital, College of Medicine, The Catholic University of Korea, Seoul, Korea
}

\section{Article history:}

Received: March 14, 2019

Revised: June 8, 2019

Accepted: July 3, 2019

\section{${ }^{*}$ Corresponding Author:}

Eun Young Kim

Division of Trauma and Surgical Critical Care, Department of Surgery, Seoul St. Mary's Hospital, College of Medicine, The Catholic University of Korea, Seoul, Korea E-mail: freesshs@naver.com

ORCID https://orcid.org/0000-0003-4408-4165

\section{ABSTRACT}

Purpose: Polymyxin B hemoperfusion (which treats septic shock by removing endotoxins of gramnegative bacteria), has been proposed as a treatment modality for intraabdominal sepsis. However, there are few studies about the factors that need to be taken into consideration for a better outcome. The aim of this study was to assess the effectiveness of polymyxin B hemoperfusion in abdominal septic shock and analyze the factors affecting the outcome of this therapy.

Methods: A retrospective review was performed in 41 patients who were diagnosed with abdominal septic shock. There were 12 patients treated with polymyxin B hemoperfusion, and 29 patients treated with conventional therapy. The clinical outcomes of the 2 groups were compared to identify the factors affecting the outcome of hemoperfusion.

Results: The decrement of vasopressor requirement represented by vasopressor dependency index was 2.5 in the hemoperfusion group and 0.1 in the conventional therapy group ( $p=0.021$ ), and inhospital mortality was $33.3 \%$ in the hemoperfusion group and $69.0 \%$ in the conventional therapy group $(p=0.045)$. In logistic regression analysis, hemoperfusion was identified as a factor reducing in-hospital mortality in patients with sufficient source control, but not in patients with insufficient source control. In patients who had undergone hemoperfusion, the longer time to initiate hemoperfusion was identified as a risk factor of in-hospital mortality $(p=0.039)$.

Conclusion: Polymyxin B hemoperfusion may be an effective therapy for treating abdominal septic shock, and early use of this modality with definite source control might be important for a better outcome.

Keywords: hemoperfusion, peritonitis, polymyxin B, septic shock

\section{Introduction}

Intraabdominal infection has been a major cause of sepsis and septic shock [1], and more than 50\% mortality can be seen in patients with abdominal sepsis unless this is managed appropriately [2]. Although the source control is a core treatment in the management of a patient with surgical peritonitis [3], additional therapies are required to salvage vital organs that have been damaged by systemic infection (due to the pathogens that have translocated into the blood stream). One of the most common types of organisms causing intraabdominal sepsis are gram negative bacteria (GNB), and the endotoxin on the outer membrane of GNB is thought to have an important role in triggering a dysregulated host response to infection and subsequent organ dysfunction, leading to research into endotoxin-targeted therapies [4]. Direct hemoperfusion with polymyxin B was proposed in 1983 as an effective treatment modality for sepsis [5].

Polymyxin $B$ is an antibiotic that has a high affinity for GNB endotoxin, and it can be immobilized onto the polystyrene 
fiber in the cartridge of the renal replacement therapy machine. By passing the patient's blood through the polymyxin $B$ immobilized fiber, endotoxin is effectively removed and sepsis can be resolved [6]. However, the factors that need to be taken into consideration when performing polymyxin $\mathrm{B}$ hemoperfusion to treat intraabdominal sepsis are debatable, especially in surgical cases such as generalized peritonitis [7].

In this study the efficacy of using polymyxin B hemoperfusion in the treatment of patients whose septic shock resulted from severe abdominal infection was assessed and the factors affecting the effectiveness of this therapy were identified.

\section{Materials and Methods}

There were 12 patients diagnosed with abdominal septic shock between December 2016 and December 2018 that underwent polymyxin $B$ hemoperfusion therapy after source control (such as bowel resection or drain insertion) at the surgical intensive care unit at St. Mary's Hospital, Seoul, Korea. The current study was approved and monitored by the St. Mary's Hospital Institutional Review Board (IRB No.: KC18RESI0293), and a retrospective medical record review for these patients (hemoperfusion group), with 29 other patients who had been treated with conventional therapy (conventional therapy group) was performed. For the diagnosis of intraabdominal infection, the laboratory test with culture studies and radiology tests, including abdominal sonogram or computed tomography scan, were performed for patients who presented with clinical signs suggestive of abdominal infection, such as abdominal tenderness, muscle guarding, or a fever over $38.00 C$. Patients were considered to be in septic shock when they showed persisting hypotension requiring vasopressors to maintain the mean arterial pressure over $65 \mathrm{mmHg}$, and had a serum lactate level over $2 \mathrm{mMol} / \mathrm{L}$ (18 mg/dL) despite the adequate volume resuscitation (according to the criteria proposed by the Third International Consensus Definitions for Sepsis and Septic Shock) [8]. In both the hemoperfusion group and the conventional therapy group, the standard intensive care for septic shock was started immediately after an abdominal septic shock diagnosis. Additionally, renal replacement therapy was also provided to patients presenting oliguria with ongoing metabolic acidosis.

To perform the polymyxin B hemoperfusion, a doublelumen venous catheter was inserted into the patient via the internal jugular vein. Then, 2 sessions of hemoperfusion using an adsorbent column containing polymyxin B (Toray Industries, Tokyo, Japan) in the renal replacement therapy machine, were performed for 2 hours (each session). Nafamostat mesilate (Torii Pharmaceuticals, Tokyo, Japan) was used as the anticoagulant for the circuit. The patient's clinical data during treatment were reviewed, and the Sepsis-related Organ Failure Assessment (SOFA) scores were assessed to evaluate the severity of organ dysfunction [9]. The inotropic score and vasopressor dependency index were also calculated to assess the severity of hemodynamic impairment. The inotropic score, the index presenting the dosage of vasoactive or vasopressor agents, was calculated.

(dopamine dose X 1) + (dobutamine dose X 1) + (adrenaline dose X 100) + (noradrenaline dose X 100) +

(phenylephrine dose X 100)

wherein all doses are expressed as $\mu \mathrm{g} / \mathrm{kg} / \mathrm{min}$ [10]. The doses of inotropic agents were continually titrated to maintain the mean arterial pressure of $65 \mathrm{mmHg}$, and the vasopressor dependency index (representing the dose-response relationship between the inotropic dose and blood pressure) was also calculated [11].

inotropic score / mean arterial pressure

Values of clinical parameters obtained at the time when the hemoperfusion had started (after initial source control) were regarded as baseline values in hemoperfusion group. Values obtained at the time when initial source control had finished, were regarded as baseline values in the conventional therapy group. Sufficiency of the initial source control was assessed in both groups because perfect elimination of the infection source may be impossible in some clinical situations. Determination of whether source control was sufficient or not was based on the patients' medical records and additional discussions with the operator surgeons were conducted when needed. In the hemoperfusion group, the time spent to obtain informed consent from the patients' relatives, and to prepare the appropriate equipment required for hemoperfusion were different in each case, so the time interval between source control and initiation of polymyxin B hemoperfusion was calculated for further analysis in all 12 patients.

\section{Statistical analysis}

Student t test or Mann-Whitney U test was used for comparison of continuous variables, and Chi-square test or Fisher's exact test was used for the comparison of proportions in categorical variables. Logistic regression analysis was performed to identify factors affecting dependent variables. Where $p<0.05$ it was considered to be statistically significant. All statistical analysis was performed using SPSS Statistics version 24.0 software (IBM, New York, NY, USA). 


\section{Results}

Demographic features and baseline hemodynamic parameters were comparable between the 2 groups, except for a higher baseline total SOFA score (hemoperfusion: $12.1 \pm 5.3$; vs conventional therapy: $8.2 \pm 3.8, p=0.012$ ) and baseline vasopressor dependency index (hemoperfusion: $2.7 \pm 3.1$; vs conventional therapy: $0.1 \pm 0.5, p=0.015$ ) in the hemoperfusion group (Table 1). The most common diagnosis was ischemic change in the colon with pan-peritonitis (hemoperfusion: 10 cases, 83.3\%; conventional therapy: 14 cases, $48.3 \%$ ), and resection of ischemic bowel was the most frequently performed operation in both groups. The comparison of treatment outcomes between the hemoperfusion group and the conventional therapy group is presented in Table 2. There was no difference in total SOFA score decrement after 72 hours between groups. However, the decrement of vasopressor requirement (represented by vasopressor dependency index), was greater in the hemoperfusion group than in the conventional therapy group (hemoperfusion: $2.5 \pm 3.1$; vs conventional therapy: $0.1 \pm 0.5, p=0.021$ ). Additionally, inhospital mortality was lower in the hemoperfusion group than in the conventional therapy group (hemoperfusion: $33.3 \%$; vs conventional therapy: $69.0 \%, p=0.045$ ).

Table 1. Patient demographics and baseline hemodynamic parameters.

\begin{tabular}{|c|c|c|c|}
\hline Variable & Hemoperfusion ( $n=12)$ & Conventional therapy $(n=29)$ & $p$ \\
\hline Age $(y)$ & $65.9 \pm 9.7$ & $66.3 \pm 11.6$ & 0.926 \\
\hline Gender (male) & $6(50.0)$ & $15(51.7)$ & 1.000 \\
\hline Baseline total SOFA score & $12.1 \pm 5.3$ & $8.2 \pm 3.8$ & 0.012 \\
\hline Baseline inotropic score & $134.2 \pm 129.9$ & $123.4 \pm 474.3$ & 0.939 \\
\hline $\begin{array}{l}\text { Baseline vasopressor dependency index } \\
(\mathrm{mmHg}-1)\end{array}$ & $2.7 \pm 3.1$ & $0.1 \pm 0.5$ & 0.015 \\
\hline Baseline lactate level (mmol/L) & $6.7 \pm 3.2$ & $5.5 \pm 5.2$ & 0.501 \\
\hline Source control sufficiency & $7(58.3)$ & $18(62.1)$ & 1.000 \\
\hline Sites of infectious source & & & 0.207 \\
\hline Stomach or duodenum & $0(0.0)$ & $2(6.9)$ & 1.000 \\
\hline Small intestine & $1(8.3)$ & $10(34.5)$ & 0.128 \\
\hline Colon or rectum & $10(83.3)$ & $14(48.3)$ & 0.079 \\
\hline Hepatobiliary & $1(8.3)$ & $3(10.3)$ & 1.000 \\
\hline
\end{tabular}

Data are presented as mean \pm SD or $n(\%)$.

SOFA $=$ Sepsis-related Organ Failure Assessment.

Table 2. Clinical outcomes and the changes of hemodynamic indices in patients.

\begin{tabular}{|c|c|c|c|}
\hline Variable & Hemoperfusion $(n=12)$ & Conventional therapy $(n=29)$ & $p$ \\
\hline Total SOFA score decrement after $72 \mathrm{~h}$ & $4.7 \pm 5.7^{*}$ & $3.9 \pm 5.4^{\dagger}$ & 0.671 \\
\hline Inotropic score decrement after $72 \mathrm{~h}$ & $123.4 \pm 129.4^{*}$ & $80.4 \pm 260.4^{\dagger}$ & 0.591 \\
\hline $\begin{array}{l}\text { Vasopressor dependency index } \\
\text { decrement after } 72 \mathrm{~h}(\mathrm{mmHg}-1)\end{array}$ & $2.5 \pm 3.1^{*}$ & $0.1 \pm 0.5^{\dagger}$ & 0.021 \\
\hline Length of mechanical ventilation (d) & $5.7 \pm 6.4$ & $4.7 \pm 7.5$ & 0.707 \\
\hline Length of ICU stay (d) & $9.5 \pm 10.6$ & $10.9 \pm 11.2$ & 0.707 \\
\hline Length of hospital stay (d) & $53.4 \pm 75.9$ & $22.6 \pm 18.7$ & 0.191 \\
\hline In-hospital mortality & $4(33.3)$ & $20(69.0)$ & 0.045 \\
\hline
\end{tabular}

Data are presented as mean \pm SD or $n(\%)$.

* Baseline value-: value at 72 hours after initiation of hemoperfusion.

† Baseline value-: value at 72 hours after initial source control.

ICU = intensive care unit; SOFA = Sepsis-related Organ Failure Assessment. 
To see whether hemoperfusion contributed to a reduced in-hospital mortality regardless of source control sufficiency patients were re-grouped depending on source control sufficiency, and logistic regression analysis was performed (Table 3). In this analysis, hemoperfusion was identified as a significant factor affecting in-hospital mortality in patients with sufficient source control (OR 0.010; $p=0.002$ ) but not in patients with insufficient source control $(p=0.223)$. Logistic regression analysis was also performed for the hemoperfusion group to determine the factors affecting in-hospital mortality in patients that had undergone hemoperfusion (Table 4). In this analysis, the longer time interval between source control and hemoperfusion initiation was identified as a risk factor of in-hospital mortality (OR 1.002; $p=0.039$ ).

\section{Discussion}

In this study, the patients with abdominal septic shock who were treated with polymyxin B hemoperfusion, had a lower in-hospital mortality than the patients treated with conventional therapy alone. There was a significant difference in the degree of vasopressor dependency index decrement between the groups, which might imply that polymyxin B hemoperfusion may be effective, especially in improving the hemodynamic aspect of a septic condition. This improvement in hemodynamics was in agreement with previous studies [11-14].
In order to maximize the effectiveness of polymyxin B hemoperfusion, the factors affecting the therapy should be identified. The observations from this study identified 2 major factors influencing the clinical outcome of hemoperfusion. Firstly, sufficient source control may be an important factor for achieving the clinical benefits of using hemoperfusion. When the patient has undergone sufficient source control, hemoperfusion has been shown to contribute to reduced in-hospital mortality. In contrast, in patients with insufficient source control, hemoperfusion has been shown to have no impact in reducing in-hospital mortality (Table 3). To understand this observation the mechanism by which hemoperfusion acts should be considered. Since the hemoperfusion device has access only to the endotoxin in the vascular space, endotoxin cannot be removed under an insufficiently source-controlled condition where the source of infection remains in extravascular structures [15]. Secondly, the time interval between source control and initiation of polymyxin B hemoperfusion may affect the clinical course of the patient. In this study, prolonged time spent before initiating hemoperfusion was shown to be a risk factor of in-hospital mortality in the patients who had undergone hemoperfusion (Table 4). Takeyama et al [16] also suggested that the time taken for polymyxin $B$ hemoperfusion to begin is associated with the clinical outcomes of septic shock patients. They demonstrated that patients who received hemoperfusion within 6 hours after the diagnosis of septic shock, had less time on ventilator support and required less catecholamine,

Table 3. Logistic regression analysis of hemoperfusion affecting in-hospital mortality according to source control sufficiency.

\begin{tabular}{cccccccc}
\hline \multirow{2}{*}{ Variable } & \multicolumn{3}{c}{ Sufficient source control } & & \multicolumn{3}{c}{ Insufficient source control } \\
\cline { 2 - 4 } & OR & CI (95\%) & $p$ & & OR & CI (95\%) & $0.431-37.108$ \\
\hline Hemoperfusion & 0.010 & $0.001-0.182$ & 0.002 & & 4.000 & 0.223 & 0.43 \\
\hline
\end{tabular}

$\mathrm{CI}=$ confidence interval; $\mathrm{OR}=$ odds ratio.

Table 4. Logistic regression analysis of factors affecting in-hospital mortality in patients who underwent hemoperfusion.

\begin{tabular}{lccc}
\hline Variable & OR & CI (95\%) & $p$ \\
\hline Age (y) & 1.081 & $0.932-1.254$ & 0.302 \\
Gender (male) & 5.000 & $0.344-72.767$ & 0.239 \\
\hline Baseline total SOFA score & 1.378 & $0.882-2.154$ & 0.159 \\
Baseline inotropic score & 1.001 & $0.992-1.011$ & 0.815 \\
\hline $\begin{array}{l}\text { Baseline vasopressor dependency index } \\
(\text { mmHg }\end{array}$ & 1.019 & $0.681-1.526$ & 0.927 \\
Baseline lactate level (mmol/L) & & & 0.969 \\
\hline Time to initiate hemoperfusion (min) & 0.992 & $0.642-1.532$ & 0.039 \\
\hline
\end{tabular}

$\mathrm{CI}=$ confidence interval; OR = odds ratio; SOFA = Sepsis-related Organ Failure Assessment. 
compared with patients who were treated 6 hours after the diagnosis of septic shock. Considering the cost burden on a patient, or limited medical resources, special ponderation should be taken on the implement of hemoperfusion especially if an unfavorable outcome is expected due to a delay in initiating hemoperfusion or there has been insufficient source control in the patient.

Although it is presumed that prerequisites such as early initiation or sufficient source control are needed for patients with intraabdominal septic shock, polymyxin B hemoperfusion may be a valuable treatment option because GNB are a major type of pathogen that are involved in peritonitis which may cause septic shock and this would be fatal in most patients. The decreased vasopressor dependency achieved by polymyxin B hemoperfusion, enables clinicians to implement other interventions that require a patient to be hemodynamically stable. This can lead to better clinical outcomes in addition to reducing the adverse effects of vasopressors. Furthermore, considering that many patients in severe septic shock require renal replacement therapy because of decreased renal function [17], polymyxin B hemoperfusion is a relatively highly available treatment because it can be readily applied by only installing an additional cartridge filter into the renal replacement therapy machine without a further hemodynamic burden to the patient.

Despite these interesting results, this study has some limitations due to the relatively small sample size and the retrospective design of the study. In addition, an endotoxin activity assay (which can quantify the severity of sepsis and assess the effectiveness of hemoperfusion numerically) was not performed in this study. However, considering that most physicians use this therapy according to the manufacturer's instructions, without evidence-based consensus about the detailed treatment protocol for polymyxin B hemoperfusion, this attempt of our study to identify the factors associated with clinical outcomes may contribute towards establishing guidelines for polymyxin B hemoperfusion treatment. Larger studies using more objective clinical parameters should be carried out to establish the optimal initiation time and exact indication of the treatment.

In conclusion, polymyxin B hemoperfusion may be a promising therapeutic modality in treating abdominal septic shock and early use of polymyxin B hemoperfusion with definite source control may be important for better outcomes.

\section{Conflicts of Interest}

The authors declare no potential conflict of interest relevant to this article.

\section{Acknowledgment}

The current study was approved and monitored by the Seoul St. Mary's Hospital Institutional Review Board (IRB No.: KC18RESI0293).

\section{References}

[1] Martin GS. Sepsis, severe sepsis and septic shock: Changes in incidence, pathogens and outcomes. Expert Rev Anti Infect Ther 2012;10(6):701-6.

[2] Angus DC, Linde-Zwirble WT, Lidicker J, Clermont G, Carcillo J, Pinsky MR. Epidemiology of severe sepsis in the United States: Analysis of incidence, outcome, and associated costs of care. Crit Care Med 2001;29(7):1303-10.

[3] Marshall JC, al Naqbi A. Principles of source control in the management of sepsis. Crit Care Clin 2009;25(4):753-68.

[4] Ronco C. Lipopolysaccharide (LPS) from the cellular wall of Gram-negative bacteria, also known as endotoxin, is a key molecule in the pathogenesis of sepsis and septic shock. Blood Purif 2014;37 Suppl 1:1.

[5] Shoji H, Tani T, Hanasawa K, Kodama M. Extracorporeal endotoxin removal by polymyxin $B$ immobilized fiber cartridge: Designing and antiendotoxin efficacy in the clinical application. Ther Apher 1998;2(1):312.

[6] Mitaka C, Tomita M. Polymyxin B-immobilized fiber column hemoperfusion therapy for septic shock. Shock 2011;36(4):332-8.

[7] Maynar J, Martinez-Sagasti F, Herrera-Gutierrez M, Marti F, Candel FJ, Belda J, et al. Direct hemoperfusion with polymyxin B-immobilized cartridge in severe sepsis due to intestinal perforation: Hemodynamic findings and clinical considerations in anticoagulation therapy. Rev Esp Quimioter 2013;26(2):151-8.

[8] Singer M, Deutschman CS, Seymour CW, Shankar-Hari M, Annane D, Bauer M, et al. The Third International Consensus Definitions for Sepsis and Septic Shock (Sepsis-3). JAMA 2016;315(8):801-10.

[9] Vincent JL, Moreno R, Takala J, Willatts S, De Mendonca A, Bruining H, et al. The SOFA (Sepsis-related Organ Failure Assessment) score to describe organ dysfunction/failure. On behalf of the Working Group on SepsisRelated Problems of the European Society of Intensive Care Medicine. Intensive Care Med 1996;22(7):707-10.

[10] Shore S, Nelson DP, Pearl JM, Manning PB, Wong H, Shanley TP, et al. Usefulness of corticosteroid therapy in decreasing epinephrine requirements in critically ill infants with congenital heart disease. Am J Cardiol 2001;88(5):591-4.

[11] Cruz DN, Antonelli M, Fumagalli R, Foltran F, Brienza N, Donati A, et al. Early use of polymyxin $B$ hemoperfusion in abdominal septic shock: The EUPHAS randomized controlled trial. JAMA 2009;301(23):2445-52.

[12] Tojimbara T, Sato S, Nakajima I, Fuchinoue S, Akiba T, Teraoka S. Polymyxin B-immobilized fiber hemoperfusion after emergency surgery in patients with chronic renal failure. Ther Apher Dial 2004;8(4):286-92.

[13] Zagli G, Bonizzoli M, Spina R, Cianchi G, Pasquini A, Anichini V, et al. Effects of hemoperfusion with an immobilized polymyxin-B fiber column on cytokine plasma levels in patients with abdominal sepsis. Minerva Anestesiol 2010;76(6):405-12.

[14] Payen DM, Guilhot J, Launey Y, Lukaszewicz AC, Kaaki M, Veber B, et al. Early use of polymyxin $B$ hemoperfusion in patients with septic shock due to peritonitis: A multicenter randomized control trial. Intensive Care Med 2015;41(6):975-84.

[15] Romaschin AD, Obiezu-Forster CV, Shoji H, Klein DJ. Novel Insights into the Direct Removal of Endotoxin by Polymyxin B Hemoperfusion. Blood Purif 2017;44(3):193-7.

[16] Takeyama N, Noguchi H, Hirakawa A, Kano H, Morino K, Obata T, et al. Time to initiation of treatment with polymyxin B cartridge hemoperfusion in septic shock patients. Blood Purif 2012;33(4):252-6.

[17] Lopes JA, Jorge S, Resina C, Santos C, Pereira A, Neves J, et al. Acute kidney injury in patients with sepsis: a contemporary analysis. Int J Infect Dis 2009;13(2):176-81. 